November 2, $2018 \quad$ 20:43 WSPC/INSTRUCTION FILE $\quad$ interf nut ${ }^{\circ} 27^{\circ} 03^{\circ} 08$

International Journal of Modern Physics D

(C) World Scientific Publishing Company

\title{
Quantum Interference Effects in Slowly Rotating NUT Space-time
}

\author{
V.S. MOROZOVA \\ Institute of Nuclear Physics and Ulugh Beg Astronomical Institute \\ Astronomicheskaya 33, Tashkent 100052, Uzbekistan \\ The Abdus Salam International Centre for Theoretical Physics, 34014 Trieste, Italy \\ moroz-vs@yahoo.com \\ B.J. AHMEDOV \\ Institute of Nuclear Physics and Ulugh Beg Astronomical Institute \\ Astronomicheskaya 33, Tashkent 100052, Uzbekistan \\ The Abdus Salam International Centre for Theoretical Physics, 34014 Trieste, Italy \\ ahmedov@astrin.uzsci.net \\ Received Day Month Year \\ Revised Day Month Year
}

\begin{abstract}
General relativistic quantum interference effects in the slowly rotating NUT space-time as the Sagnac effect and the phase shift effect of interfering particle in neutron interferometer are considered. It was found that in the case of the Sagnac effect the influence of NUT parameter is becoming important due to the fact that the angular velocity of the locally non rotating observer must be larger than one in the Kerr space-time. In the case of neutron interferometry it is found that due to the presence of NUT-parameter an additional term in the phase shift of interfering particle emerges. This term can be, in principle, detected by sensitive interferometer and derived results can be further used in experiments to detect the gravitomagnetic charge. Finally, as an example, we apply the obtained results to the calculation of the UCN (ultra-cold neutrons) energy level modification in the slowly rotating NUT space-time.
\end{abstract}




\section{Introduction}

Due to the weakness of gravitational field in the Solar system, many effects of general relativity predicted by theory are tiny and not detected yet. Among them theoretical prediction about existence of gravitomagnetic monopolar charge which was theoretically discovered by Newman, Tamburino and Unti 1 and called as NUT parameter.

According to Gravitoelectromagnetism 2 there is analogy between gravitation and electromagnetism in weak field and slow motion approximation. As electric currents in classical electrodynamics create magnetic field, in the same way mass-energy currents must produce gravitomagnetic fields. Verification of the gravitomagnetism would test and prove the ideas of Einstein and others that the local inertial frames are determined by the distribution and currents of mass-energy throughout the Universe. For some relevant information about research in this direction one can look to 20.5].

Moreover similar to magnetic Dirac's monopole there is hypothesis about existence of gravitomagnetic monopole. Here we use slowly rotating NUT metric for the exterior space-time of source with nonvanishing NUT-parameter, related with gravitomagnetic charge, as we did in our preceding papers 6 6] In this metric space-time we investigate two quantum interference phenomena: so called Sagnac effect and the phenomena of phase shift in neutron interferometer. It is connected with the fact that quantum interference measurements based on macroscopic quantum effects can provide sensitive precise accuracy to detect weak gravitational effects. Our main goal here is to find influence, caused by the existence of the NUT-parameter, on these effects and prepare proposals for further experiments, which could detect this influence.

The Sagnac effect is well known and thoroughly studied (see, for example 8 ). It presents the fact that between light or matter beam counter-propagating along a closed path in a rotating interferometer a fringe shift $\Delta \varphi$ arises. This phase shift can be interpreted as a time difference between the propagation times of the co-rotating and counter-rotating beams. The expression for time delay $\Delta T$ between two beams, as it can be seen below, doesn't include the mass or energy of particles. That is why we may consider the Sagnac effect as the "universal" effect of the geometry of space-time, independent of the physical nature of the interfering beams. Here we extend the recent results obtained in the papers $9 \mid 10$, where it has been shown a way of calculation of this effect in analogy with the Aharonov-Bohm effect, to the case of slowly rotating NUT space-time.

In addition to this technique we use here one more alternate way to calculate the Sagnac effect, which is based on the idea suggested in works 11112 that the speed of light depends on the direction of a light ray in space around axial-symmetric gravitating object. Thus, we can explain the time difference between two counter-propagating beams as a result of the difference in speed in the different directions. We will use this way to calculate the time delay and compare result with the one already obtained by the previous method.

The experiment to test the effect of the gravitational field of the Earth on the phase shift in a neutron interferometer were first proposed by Overhauser and Colella 13. Then this experiment was successfully performed by Collela, Overhauser and Werner 14. After that, there were found other effects, related with the phase shift of interfering particles. Among them the effect due to the rotation of the Earth (the Coriolis force) 15116, which is the quantum mechanical analog of the Sagnac effect, and the Lense-Thirring effect 17, which is a general relativistic effect due to the dragging of the reference frames. Particle interferometry in Plebański-Demiański generalized black hole space-time is presented in the recent work $\underline{18}$. Interesting effects, caused by including the effects of particle's spin, or terms violating the Einstein Equivalence Principle can be found in 1922 . 
In the paper 23 a unified way of study of the effects of phase shift in neutron interferometer due to the various phenomena was proposed. Here we extend this formalism to the case of the slowly rotating NUT space-time in order to derive such phase shift due to the existence of NUT-parameter.

Recently Granit experiment 24 verified the quantization of the energy level of ultra-cold neutrons (UCN) in the Earth's gravity field and new, more precise experiments are planned to be performed. Experiments with UCN have high accuracy and that is the reason to look for verification of the gravitomagnetism in such experiments. As an example we investigate modification of UCN energy levels caused by the existence of NUT parameter.

Throughout we use a space-like signature $(-,+,+,+)$, Greek indices are taken to run from 0 to 3 , Latin indices run from 1 to 3 .

The Kerr-NUT spacetime metric for the gravitomagnetic monopole in the approximation of slowly rotating and weak gravitating source looks as (see 25 )

$$
d s^{2}=-N^{2} d t^{2}+N^{-2} d r^{2}+r^{2}\left(d \theta^{2}+\sin ^{2} \theta d \phi^{2}\right)-2\left[\omega(r) r^{2} \sin ^{2} \theta+2 l N^{2} \cos \theta\right] d t d \phi,
$$

where $N^{2}=(1-2 M / r)$ is the lapse function, $M$ is the total mass of the gravitating object, $\omega=2 M a / r^{3}$ is the angular velocity of dragging of inertial frames, $a=J / M$ is the specific angular momentum being equal to the total momentum $J$ of the gravitating object per unit mass, $l$ is the NUT-parameter (we assume that $c=G=1$, where $G$ is the Newtonian gravitational constant, $c$ is the speed of light).

The paper is organized as follows. In section 2 we calculate the Sagnac effect in the slowly rotating NUT space-time. In section 3 we find the expression for the phase shift in neutron interferometer and UCN energy levels change caused by the existence of NUT parameter. We also investigate the derived expressions and compare them with already known terms. Section 4 is devoted to conclusions.

\section{The Sagnac effect}

As it was presented in the paper 10 the Sagnac effect can be calculated in analogy with the Aharonov-Bohm effect. In this case the phase shift between two light or matter beams counter-propagating in flat space-time along a closed path in a rotating interferometer will be

$$
\Delta \varphi=\frac{2 m u_{0}}{c \hbar} \oint_{C} \mathbf{A}_{G} \cdot d \mathbf{x} .
$$

The corresponding time difference between the propagation times of the co-rotating and counter-rotating beams will be equal to

$$
\Delta T=\frac{2 u_{0}}{c^{3}} \oint_{C} \mathbf{A}_{G} \cdot d \mathbf{x} .
$$

In the formulae (2) and (3) $m$ is the mass (or the energy) of the particle of the interfering beams, $\mathbf{A}_{G}$ is the gravito-magnetic vector potential, which is obtained from the expression

$$
\mathbf{A}_{i}^{G} \equiv c^{2} \frac{u_{i}}{u_{0}},
$$

and $u(x)$ is the unit four-velocity of particles:

$$
u^{\alpha} \equiv\left\{\frac{1}{\sqrt{-g_{00}}}, 0,0,0\right\}, \quad u_{\alpha} \equiv\left\{-\sqrt{-g_{00}}, g_{i 0} u^{0}\right\} .
$$


Phase shift in the equation (2) is detected by a uniformly rotating interferometer and the time difference in (3) is measured by comoving observers.

Now we apply these equations to make the relevant calculations in spacetime metric (11). It should be noticed that in the equatorial plane $(\theta=\pi / 2)$ influence of the NUT-part of the metric to the effect will be zero. To see how the Sagnac time-delay and phase shift will be changed due to presence of NUT-parameter, let us consider the plane, where $\theta=\pi / 4$, so $\sin ^{2} \theta=1 / 2$ and $\cos \theta=1 / \sqrt{2}$.

After the coordinate transformation $\phi \rightarrow \phi+\Omega t$, where $\Omega$ is the angular velocity of the gravitating object the line element will look as

$$
\begin{aligned}
d s^{2} & =-\left(N^{2}-\frac{r^{2} \Omega^{2}}{2}+\Omega \omega r^{2}+2 \sqrt{2} l N^{2} \Omega\right) d t^{2}+N^{-2} d r^{2}+\frac{r^{2}}{2} d \phi^{2} \\
& +2\left(\frac{r^{2}(\Omega-\omega)}{2}-\sqrt{2} l N^{2}\right) d \phi d t .
\end{aligned}
$$

From this equation one can immediately see, that the unit vector field $u(x)$ along the trajectories $r=$ $R=$ const will be

$$
\begin{aligned}
& u_{0}=-\left(u^{0}\right)^{-1}, \\
& u_{\phi}=\left(\frac{R^{2}(\Omega-\omega)}{2}-\sqrt{2} l N^{2}\right) u^{0},
\end{aligned}
$$

where a notation

$$
u^{0}=\left(N^{2}-\frac{R^{2} \Omega^{2}}{2}+\Omega \omega R^{2}+2 \sqrt{2} l N^{2} \Omega\right)^{-1 / 2}
$$

is introduced.

Now, inserting the components of $u(x)$ into the equation (4) one obtain

$$
\mathbf{A}_{\phi}^{G}=-\left(\frac{R^{2}(\Omega-\omega)}{2}-\sqrt{2} l N^{2}\right)\left(u^{0}\right)^{2} .
$$

Integrating vector potential, as it is shown in equations (2) and (3), one can get the following expressions for $\Delta \varphi$ and $\Delta T$ (here we returned to the physical units):

$$
\begin{gathered}
\Delta \varphi=\frac{4 \pi m}{\hbar}\left(\frac{R^{2}(\Omega-\omega)}{2}-\sqrt{2} c l N^{2}\right)\left(u^{0}\right)^{2}, \\
\Delta T=\frac{4 \pi}{c^{2}}\left(\frac{R^{2}(\Omega-\omega)}{2}-\sqrt{2} c l N^{2}\right)\left(u^{0}\right)^{2} .
\end{gathered}
$$

Following to the paper 10 one can find a critical angular velocity $\bar{\Omega}$

$$
\bar{\Omega}=\omega+\frac{2 \sqrt{2} c l N^{2}}{R^{2}},
$$

which corresponds to zero time delay $\Delta T=0 . \bar{\Omega}$ is the angular velocity of the zero angular momentum observers (ZAMO). As we can see, the term with NUT-parameter presents a positive addition to this velocity, in other words, parameter $\bar{\Omega}$ in Kerr-NUT space-time becomes larger with compare to that in Kerr one.

Alternate approach for interpretation of Sagnac effect is given in the papers 11, 12, which is based on anisotropy of the speed of light. According to Einstein the definition of simultaneity can be derived from 
the postulate of the constancy of the speed of light. However the constancy and anisotropy of the speed of light is not obvious, when one consider light propagation in arbitrary four-dimensional Riemann space-time in general relativity. Moreover assumption about the anisotropy of the speed of light is not obvious even in inertial frames, because all laboratory methods of measuring the speed of light establish anisotropy only then light is moving "there and back" rather than only "there" or "back". Measurement of the speed of light in one single direction requires the establishment of the way to compare time intervals in different points of space before making a measurement i.e. the definition of simultaneity without including any knowledge about the speed of light. Therefore the definition of simultaneity is primary and underlie any physical measurement, whereas the value of the speed of light is secondary and derived from this fundamental definition.

Let $P_{1}$ and $P_{2}$ be the events on the world line of the clock being at rest in the given frame, corresponding to emission and registration of the light signal reflected (event $P^{\prime}$ ) from the mirror placed at slight distance from the clock. Each of these events have fixed value of proper time $\tau\left(P_{1}\right), \tau\left(P_{2}\right)$ and $\tau\left(P^{\prime}\right)$.

According to 12 an event $P$ being simultaneous to $P^{\prime}$ is defined as the event, moment of proper time of which satisfies to the condition

$$
\tau(P)=\frac{1}{2}\left[\tau\left(P_{1}\right)+\tau\left(P_{3}\right)\right]+\frac{1}{2}\left[\tau\left(P_{1}\right)-\tau\left(P_{3}\right)\right] \frac{a_{n} d x^{n}}{\sqrt{\left(g_{i k}-\frac{g_{0 i} g_{0 k}}{g_{00}}\right) d x^{i} d x^{k}}},
$$

while Einstein's definition of simultaneity is

$$
\tau(P)=\frac{1}{2}\left[\tau\left(P_{1}\right)+\tau\left(P_{3}\right)\right]
$$

In equation (13) $a_{n}$ are the covariant components of the metric vector, defined as

$$
a_{n}=\frac{g_{0 n}}{\sqrt{-g_{00}}}, a=\sqrt{\frac{g^{i k} a_{i} a_{k}}{1-g^{m j} a_{m} a_{j}}} .
$$

Using equation (13) one can obtain expression for interval of proper time between two arbitrary, but close to each other events as

$$
d \tau=\sqrt{-g_{00}}\left(d x^{0}+\frac{g_{0 i}}{g_{00}} d x^{i}\right)+a_{i} d x^{i}
$$

and write the metric of space-time in the form

$$
d s^{2}=-e^{-2 \phi} d t^{2}+2 e^{-\phi} a_{i} d x^{i} d t+d l^{2},
$$

where

$$
\begin{gathered}
d l^{2}=h_{i k} d x^{i} d x^{k}, \\
h_{i k}=g_{i k}-\frac{g_{0 i} g_{0 k}}{g_{00}}-a_{i} a_{k} .
\end{gathered}
$$

From equation (17) one can derive the expression for the absolute value of the speed of light as

$$
v_{c}=\sqrt{-g_{00}}\left(-a \cos \alpha+\sqrt{1+a^{2} \cos ^{2} \alpha}\right),
$$

where $\alpha$ is the angle between the light ray and the metric vector. Consequently, the speed of light has the smallest value when the light ray propagates in the direction along the metric vector, and the largest value in the opposite direction. The speed of light is constant in all directions, lying on the conical surface with 
the top in a given point and the axis of symmetry along the metric vector. From the equation (20) one can see that the product of the values of the speed of light in the opposite direction is constant in a given point and the following equation takes place

$$
\frac{1}{-g_{00}} \cdot v_{c} \bar{v}_{c}=1
$$

where $\bar{v}_{c}$ is the absolute value of the vector $\left(-\vec{v}_{c}\right)$.

Using these discourses, one can conclude that the time difference between the counter-propagating light rays on a closed path is equal to the integral

$$
\Delta t=\oint\left(\frac{1}{v_{c}}-\frac{1}{\bar{v}_{c}}\right) d l
$$

where $d l$ is the element of the path.

In axial symmetric space-times (in particular in one of the slowly rotating source with the non-zero NUTparameter) metric vector is perpendicular to the axis of symmetry. For the light rays, counter-propagating in the plane $\theta=\pi / 4$, one should choose the angles $\alpha$ being equal to 0 and $\pi$ and

$$
v_{c}(\alpha=\pi, 0)=\sqrt{-g_{00}}\left( \pm a+\sqrt{1+a^{2}}\right) .
$$

Making algebraic transformations in the equation (22) with help of equations (21) and (23) one can find

$$
\Delta t=\oint \frac{\bar{v}_{c}-v_{c}}{v_{c} \bar{v}_{c}} d l=\oint \frac{\left(\bar{v}_{c}-v_{c}\right)}{-g_{00}} d l=2 \oint u^{0} a d l
$$

In the space-time metric (6) the single nonvanishing component of the metric vector (15) is

$$
a_{3}=u^{0}\left(\frac{r^{2}(\Omega-\omega)}{2}-\sqrt{2} l N^{2}\right)
$$

and its absolute value is

$$
a=\frac{\frac{\sqrt{2} u^{0}}{r}\left(\frac{r^{2}(\Omega-\omega)}{2}-\sqrt{2} l N^{2}\right)}{\left(1-\frac{2\left(u^{0}\right)^{2}}{r^{2}}\left(\frac{r^{2}(\Omega-\omega)}{2}-\sqrt{2} l N^{2}\right)^{2}\right)^{1 / 2}},
$$

which in the linear approximation in angular velocity of rotation and NUT-parameter takes simple form

$$
a=\frac{\sqrt{2} u^{0}}{r}\left(\frac{r^{2}(\Omega-\omega)}{2}-\sqrt{2} l N^{2}\right) .
$$

Inserting it into (24) we eventually obtain an expression for the time delay between two counterpropagating rays in the Sagnac effect (in physical units)

$$
\Delta t=\frac{\sqrt{2} \cdot 4 \pi}{c^{2}}\left(\frac{R^{2}(\Omega-\omega)}{2}-\sqrt{2} c l N^{2}\right)\left(u^{0}\right)^{2} .
$$

Now we would like to underline that the Sagnac effect here is calculated in the plane $\theta=\pi / 4$ instead of traditional one $\theta=\pi / 2$ in order to find effect related to NUT-parameter which vanishes in the equatorial plane $\theta=\pi / 2$. Classical experiment on Sagnac effect has been performed in the equatorial plane being 
orthogonal to axis of rotation where both approaches given by equations (11) and (28) would give common result

$$
\Delta T=\frac{4 \pi R^{2} \Omega}{c^{2}}\left(u^{0}\right)^{2} .
$$

Therefore the difference between equations (11) and (28) is due to calculations made in the plane $\theta=\pi / 4$.

It should be noticed that in equations (11) and (28) the term arising from the Lense-Thirring effect has spatial dependence as $1 / r$, while the term produced by non-zero NUT-parameter has no spatial dependence and will not decay with the distance from the surface of the gravitating object.

\section{Phase shift}

Following to the discourses suggested in the work 23 we start from the covariant Klein-Gordon equation

$$
\nabla^{\mu} \nabla_{\mu} \Phi-(m c / \hbar)^{2} \Phi=0
$$

define the wave function $\Phi$ of interfering particles as

$$
\Phi=\Psi \exp \left(-i \frac{m c^{2}}{\hbar} t\right)
$$

and neglect the terms of order $O\left((v / c)^{2}\right)$.

Using slowly rotating NUT metric (1) and making the coordinate transformation $\phi \rightarrow \phi-\Omega t$ we obtain the following expression for the Schrödinger equation

$$
i \hbar \frac{\partial \Psi}{\partial t}=-\frac{\hbar^{2}}{2 m}\left[\frac{1}{r^{2}} \frac{\partial}{\partial r}\left(r^{2} \frac{\partial}{\partial r}\right)-\frac{L^{2}}{r^{2} \hbar^{2}}\right] \Psi-\frac{M m}{r} \Psi-\Omega L_{z} \Psi+\frac{2 M a}{r^{3}} L_{z} \Psi+\frac{l \cos \theta}{r^{2} \sin ^{2} \theta} L_{z} \Psi,
$$

where $L^{2}, L_{z}$ are the orbital angular momentum operators:

$$
\begin{gathered}
L^{2}=-\hbar^{2}\left[\frac{1}{\sin \theta} \frac{\partial}{\partial \theta}\left(\sin \theta \frac{\partial}{\partial \theta}\right)+\frac{1}{\sin ^{2} \theta} \frac{\partial^{2}}{\partial \phi^{2}}\right], \\
L_{z}=-i \hbar \frac{\partial}{\partial \phi} .
\end{gathered}
$$

From the equation (32) one can see that the Hamiltonian of the particle in interferometer can be represented as a sum

$$
H=H_{0}+H_{1}+H_{2}+H_{3}+H_{4}
$$

where

$$
H_{0}=-\frac{\hbar^{2}}{2 m} \frac{1}{r^{2}} \frac{\partial}{\partial r}\left(r^{2} \frac{\partial}{\partial}\right)+\frac{L^{2}}{2 m r^{2}}, \quad H_{1}=-\frac{M m}{r}, \quad H_{2}=-\Omega L_{z}, \quad H_{3}=\frac{2 M a}{r^{3}} L_{z} .
$$

$H_{0}$ is the Hamiltonian for a freely propagating particle, $H_{1}$ is the Newtonian gravitational potential energy, $H_{2}$ is concerned to the rotation of the gravitating source, $H_{3}$ is related to the Lense-Thirring effect (dragging of the inertial frames). The phase shift terms due to $H_{1}, H_{2}$ and $H_{3}$ are

$$
\begin{aligned}
& \beta_{\text {grav }}=\frac{m^{2} g S \lambda}{2 \pi \hbar^{2}} \sin \phi, \quad \beta_{\text {rot }} \simeq \frac{2 m \vec{\Omega} \cdot \mathbf{S}}{\hbar}, \\
& \beta_{\text {drag }} \simeq \frac{2 m}{\hbar R^{3}} \mathbf{J} \cdot\left[\mathbf{S}-3\left(\frac{\mathbf{R}}{R} \cdot \mathbf{S}\right) \frac{\mathbf{R}}{R}\right],
\end{aligned}
$$


correspondingly.

Here $S=d_{1} d_{2}$ is the area of interferometer, $\mathbf{S}$ is the area vector of the sector ABCD (see fig. 1), $\vec{\Omega}=(0,0, \Omega)$ and $\mathbf{J}=(0,0, J)$ are the angular velocity and the angular momentum vectors of the object correspondingly, $\mathbf{R}$ is the position vector of the instrument from the center of the gravitating object, $\lambda$ is de Broglie wavelength.

The last term of the equation (32) represents the part $H_{4}$ of Hamiltonian

$$
H_{4}=\frac{l \cos \theta}{r^{2} \sin ^{2} \theta} L_{z}
$$

related to the NUT-parameter.

Integrating it over time along the trajectory of the particle, one can find the corresponding phase shift

$$
\beta_{4}=\frac{1}{\hbar} \int \frac{l \cos \theta}{r^{2} \sin ^{2} \theta} L_{z} d t .
$$

Using a unit vector $\mathbf{n}=(0,0,1)$, presenting $\mathbf{r}=\mathbf{R}+\mathbf{r}$ ', where $\mathbf{r}^{\prime}$ denotes position of the given point of the interferometer from the center of the instrument, and assuming that $\mathbf{r} / \mathbf{R}$ is small one can obtain that the angular dependence of $\beta_{N U T}$

$$
\begin{aligned}
\beta_{N U T} & =\beta_{4(A B D)}-\beta_{4(A C D)}=-\frac{l m \cos \theta}{\hbar \sin ^{2} \theta} \oint \frac{\mathbf{n} \cdot(\mathbf{r} \times d \mathbf{r})}{r^{2}} \\
& =-\frac{l m \cos \theta}{\hbar R^{2} \sin ^{2} \theta} \mathbf{n} \oint \frac{\left(\mathbf{R}+\mathbf{r}^{\prime}\right) \times d \mathbf{r}^{\prime}}{\left|\mathbf{R}+\mathbf{r}^{\prime}\right|^{2}} \simeq-\frac{l m \cos \theta}{\hbar R^{2} \sin ^{2} \theta}\left[\mathbf{S}-2\left(\frac{\mathbf{R}}{R} \cdot \mathbf{S}\right) \frac{\mathbf{R}}{R}\right] \mathbf{n}
\end{aligned}
$$

has two singularities at $\theta=0$ and $\theta=\pi / 2$. In further calculations we assume $\theta=\pi / 4$ and use for the expression for angular dependence $\cos \theta / \sin ^{2} \theta$ its value $\sqrt{2}$.

Now we would compare this result with other terms, derived in the paper 23 in order to evaluate its magnitude. As it was found, $\beta_{\text {drag }}$ is the smallest term between others, which has the similar structure as our $\beta_{N U T}$ and $10^{9}$ times smaller then $\beta_{\text {rot }}$. But, in principle, it can be detected with a sensitive "figureeight" interferometer due to its dependence on the distance from the center of the Earth as well as the size and direction of interferometer (see 26 ). Thus, it is reasonable to consider the ratio $\beta_{N U T} / \beta_{\text {drag }}$. If $\mathbf{R}$ is perpendicular to $\mathbf{A}$ then (here we returned to physical units)

$$
\frac{\beta_{N U T}}{\beta_{\text {drag }}}=\frac{5 \sqrt{2}}{2} \frac{l c^{3}}{G M R \Omega} .
$$

For the Earth parameters $\Omega \sim 10^{-5} s^{-1}, M \approx 5.96 \times 10^{27} \mathrm{~g}$ and $R \approx 6.4 \times 10^{8} \mathrm{~cm}$ one can obtain

$$
\frac{\beta_{N U T}}{\beta_{d r a g}} \sim 10^{7} l
$$

which shows that detectable value of gravitomagnetic charge should be at least $l \sim 10^{6} \mathrm{~cm}$.

Now we apply the obtained result for the Hamiltonian of the particle, moving in a rotating space-time, endowed with the gravitomagnetic charge, to the calculation of energy level of ultra-cold neutrons (UCN) (as it was done for slowly rotating Kerr space-time in the work 27 ). In the paper 27 the effect of the angularmomentum perturbation of the Hamiltonian $H_{2}=-\Omega L_{z}$ on the energy levels of UCN is investigated. We want to generalize this correction to the case of the gravitating object (Earth in particular case) which 


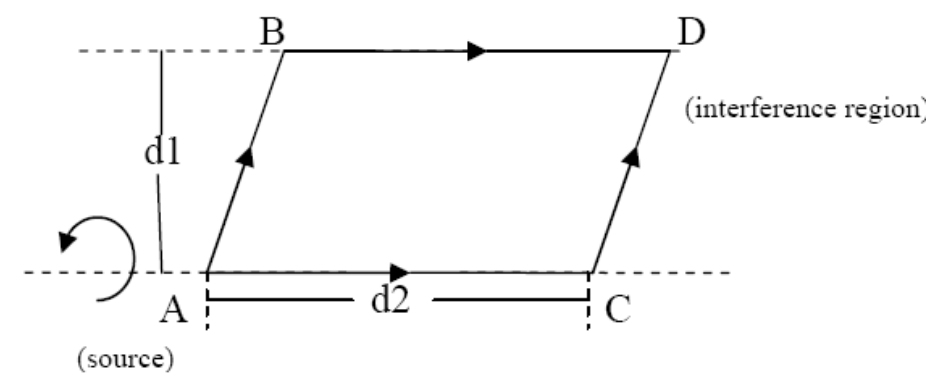

Fig. 1. Schematic illustration of alternate paths separated in vertical direction in a neutron interferometer.

possess also the NUT parameter. Denote as $\psi$ the unperturbed non-relativistic stationary state of the 2spinor (describing UCN) in the field of the rotating gravitating object with non-zero NUT-parameter. Then we have

$$
H_{4} \psi=-i \hbar \frac{l \cos \theta}{r^{2} \sin ^{2} \theta} \frac{\partial \psi}{\partial \phi}=-i \hbar \frac{l \cos \theta}{r \sin \theta} \nabla \psi \cdot \mathbf{e}_{\phi},
$$

where in the spherical coordinates Laplacian $\nabla \psi$ is

$$
\nabla \psi=\frac{\partial \psi}{\partial r} \mathbf{e}_{r}+\frac{1}{r} \frac{\partial \psi}{\partial \theta} \mathbf{e}_{\theta}+\frac{1}{r \sin \theta} \frac{\partial \psi}{\partial \phi} \mathbf{e}_{\phi}
$$

Adopting new Cartesian coordinates $x, y, z$ with $\mathbf{e}_{x} \equiv \mathbf{e}_{\phi}$ and axis $z$ being local vertical, when the stationary state is assumed to have the form

$$
\phi(\mathbf{x})=\phi_{v}(z) e^{i\left(k_{1} x+k_{2} y\right)},
$$

one can derive from (43)

$$
H_{4} \psi=-i \hbar \frac{l \cos \theta}{r \sin \theta} \frac{\partial \psi}{\partial x}=\hbar k_{1} \frac{l \cos \theta}{r \sin \theta} \psi=m u_{1} \frac{l \cos \theta}{r \sin \theta} \psi,
$$


with

$$
u_{1} \equiv \mathbf{u} \cdot \mathbf{e}_{\phi}, \quad \mathbf{u} \equiv \hbar\left(k_{1} \mathbf{e}_{x}+k_{2} \mathbf{e}_{y}\right) / m
$$

Following to the work 27 we can compute modification of the energy level as a first-order perturbation:

$$
(\delta E)_{N U T} \simeq\left(\psi \mid H_{4} \psi\right)=m u_{1} \int \frac{l \cos \theta}{r \sin \theta}|\psi|^{2} d V .
$$

Assume $r=(R+z) \cos \chi$ (where $\chi$ is the latitude angle) and $\cos \theta / \sin \theta$ to be equal to 1 , that is $\theta=\pi / 4$. Assuming now $z \ll R$ one can extend (48) as

$$
(\delta E)_{N U T} \simeq m u_{1} \frac{l}{R \cos \chi} \int\left(1-\frac{z}{R}\right)|\psi|^{2} d V=m u_{1} \frac{l}{R \cos \chi}-m u_{1} \frac{l}{R^{2} \cos \chi} \int z|\psi|^{2} d V .
$$

Then we remember that $\int z|\psi|^{2} d V$ is the average value $<z>_{n}$ of $z$ for the stationary state $\psi=\psi_{n}$. For further calculations we need to use formulae for $\langle z\rangle_{n}$ from 27

$$
<z>_{n}=\frac{2}{3} \frac{E_{n}}{m g}
$$

Now one can easily estimate the relative modification of the energy level $E_{n}$ of the neutrons, placed in gravitomagnetic field, as

$$
\frac{(\delta E)_{N U T}}{E_{n}} \simeq-\frac{2 u_{1} l}{3 g \cos \chi R^{2}}
$$

We numerically estimate the obtained modification using the following parameters for the Earth: $u_{1} \simeq$ $+10 \mathrm{~m} / \mathrm{s}, l \sim 10^{3} \mathrm{~cm}, \cos \chi \simeq 0.71, g \simeq 10 \mathrm{~m} / \mathrm{s}^{2}$ and $R \simeq 6.4 \times 10^{8} \mathrm{~cm}$. Then

$$
\frac{(\delta E)_{N U T}}{E_{n}} \simeq 2.2 \times 10^{-15} .
$$

From this one can see, that the influence of NUT parameter will be stronger in the vicinity of compact gravitating objects with small $R$.

\section{Conclusions}

In the present paper we have considered quantum interference effects in slowly rotating Kerr-NUT spacetime and found that the presence of NUT-parameter in the metric can have influence on different quantum phenomena. Namely, we obtained the phase shift and time delay in Sagnac effect can be affected by monopolar gravitomagnetic charge. Then, we found an expression for the phase shift in a neutron interferometer due to existence of NUT-parameter and concluded that it can be detected with the help of "figure-eight" interferometer. We also investigated the application of obtained result to the calculation of energy levels of UCN and found modifications to be rather small for the Earth, but maybe more relevant for compact astrophysical objects. Obtained information can be further used in experiments to detect the interference effects related to the phenomena of gravitomagnetism. 
November 2, 2018 20:43 WSPC/INSTRUCTION FILE interf nut $27^{\circ} 03^{*} 08$

\section{Acknowledgments}

VSM thanks the IUCAA for warm hospitality during her stay in Pune and AS-ICTP for the travel support. This research is also supported in part by the UzFFR (projects 5-08 and 29-08) and projects FA-F2-F079, FA-F2-F061 and A13-226 of the UzAS. This work is partially supported by the ICTP through the OEAPRJ-29 project and the Regular Associateship grant. BJA acknowledges the partial financial support from NATO through the reintegration grant EAP.RIG.981259.

\section{References}

1. E. Newman, L. Tamburino and T. Unti, J. Math. Phys. 4, 915 (1963).

2. I. Ciufolini and J.A. Wheeler, Gravitation and Inertia (Princeton University Press, 1993).

3. D. Lynden-Bell and M. Nouri-Zonoz, Rev. Mod. Phys. 70, 427 (1998).

4. D. Bini, C. Cherubini, R.T. Jantzen and B. Mashhoon, Class. Quantum Grav. 2, 457 (2003).

5. V.S. Manko and E. Ruiz, Class. Quantum Grav. 22, 3555 (2005).

6. V. Kagramanova and B. Ahmedov, Gen.Rel.Grav. 38, 823 (2006).

7. A.A. Abdujabbarov, B.J. Ahmedov and V.G. Kagramanova, Gen.Rel.Grav. 40, (2008) in press, arxiv: 0802.4349v2 [gr-qc].

8. G. Rizzi and M.L. Ruggiero, in Relativity in Rotating Frames, eds. G. Rizzi and M.L. Ruggiero, in the series "Fundamental Theories of Physics", Kluwer Academic Publishers, Dordrecht (2004), gr-qc/0305084.

9. G. Rizzi and M.L. Ruggiero, Gen. Rel. Grav. 35, 1743 (2003).

10. M.L. Ruggiero, Gen. Rel. Grav. 37, 1845 (2005).

11. L.Ya. Arifov and N.S. Bespalova, Zh. Exp. Teor. Fiz. 58, 568 (1970) in Russian [English translation is in Sov. Phys. JETP].

12. L.Ya. Arifov, Doklady Akad. Nauk SSSR 210, 1320 (1973), in Russian.

13. A.W. Overhauser and R. Colella, Phys. Rev. Lett 33, 1237 (1974).

14. R. Colella, A.W. Overhauser and S.A. Werner, Phys. Rev. Lett. 34, 1472 (1975).

15. L.A. Page, Phys. Rev. Lett. 35, 543 (1975).

16. S.A. Werner, J.-L. Staudenmann and R. Colella, Phys. Rev. Lett. 42, 1103 (1979).

17. B. Mashhoon, F.W. Hehl and D.S. Theiss, Gen. Rel. Grav. 16, 711 (1984).

18. V. Kagramanova, J. Kunz and C. Lämmerzahl, Class. Quantum Grav. 25, (2008) in press, arxiv: 0801.4514v2 [gr-qc].

19. J. Audretsch and C. Lämmerzahl, J. Phys. A: Math. Gen. 16, 2475 (1983).

20. Ch. J. Borde, J.-C. Houard, and A. Karasiewicz, in Gyros, Clocks, and Interferometers: Testing Relativistic Gravity in Space, eds. C. Lämmerzahl, C.W.F. Everitt, and F.W. Hehl, SpringerVerlag, Berlin, page 403 (2001).

21. Ch. J. Borde, C. R. Acad. Sci. Paris Serie IV 2, 509 (2001).

22. C. Lämmerzahl, Class. Quantum Grav. 14, 13 (1998).

23. J. Kuroiwa, M. Kasai and T. Futamase, Phys. Lett. A 182, 330 (1993).

24. V. V. Nesvizhevsky et. al., Phys. Rev. D 67, 102002 (2003).

25. D. Kramer, H. Stephani, M. Maccallum and E. Herlt, Exact Solutions of the Einsteins Field Equations (Deutscher Verlag der Wissenschaften, Berlin, 1980).

26. J. Anandan and R.Y. Chiao, Gen. Rel. Grav. 14, 515 (1982).

27. M. Arminjon, Phys. Let. A 372, 2196 (2008). 